\title{
On the Quantification of Aging Effects on Biometric Features
}

\author{
Andreas Lanitis and Nicolas Tsapatsoulis \\ School of Communication and Applied Arts, Cyprus University of Technology, \\ P.O. Box 50329, 3036, Lemesos, Cyprus \\ \{andreas.lanitis, nicolas.tsapatsoulis\}@cut.ac.cy
}

\begin{abstract}
Biometric templates are often used in intelligent human computer interaction systems that include automated access control and personalization of user interaction. The effectiveness of biometric systems is directly linked with aging that causes modifications on biometric features. For example the long term performance of person identification systems decreases as biometric templates derived from aged subjects may display substantial differences when compared to reference templates whereas in age estimation, aging variation allows the age of a subject to be estimated. In this paper we attempt to quantify the effects of aging for different biometric modalities facilitating in that way the design of systems that use biometric features. In this context the homogeneity of statistical distributions of biometric features belonging to certain age classes is quantified enabling in that way the definition of age sensitive and age invariant biometric features. Experimental results demonstrate the applicability of the method in quantifying aging effects.
\end{abstract}

Keywords: Biometric Templates, Aging Variation, Intelligent Human Computer Interaction.

\section{Introduction}

Intelligent Human Computer Interaction (HCI) systems with abilities to adapt to individual needs of different users have received increased attention in the research community [9]. In most cases the adaptation of interaction mode is accomplished based on the preferences set by a user. As an alternative, automatic adaptation of the way that a user interacts with a machine can be achieved by analyzing biometrical features of a user. Among other possible scenarios biometric features can be used for identifying the user of a machine allowing in that way automatic access and selection of user preferences and estimating the age of a user enabling the implementation of ageadaptive interaction systems.

Adaptive HCI based on biometric templates is heavily depended on the suitability of features used for the intended application. For example in the case of user authentication discriminatory biometric features invariant to facial expression, face orientation, lighting and aging need to be used. In general intelligent methods are required for selecting the most appropriate biometric features according to the application. 
A key issue related to the use of biometric templates is aging variation that causes modifications in biometric features [16]. Despite the fact that aging effects need to be taken into account during the design of biometric systems, so far the topic of assessing the aging invariance of biometric templates did not receive much attention. In this paper we attempt to quantify the effect of aging on different biometric templates. In this context we formulate a metric; called the 'Aging Impact' that reflects the expected intensity of aging effects for different biometric templates. High values of the Aging Impact indicate that the corresponding biometric features are subjected to intense aging-related appearance. According to the application where a biometric feature is intended to be used it may be desirable to select biometric features that exhibit low Aging Impact (i.e. for person identification applications) or high Aging Impact (i.e. for age estimation applications). As part of our study we estimate the Aging Impact for biometric templates of different types and different modalities such as faces, fingerprints, palms, hand movements and face movements. To the best of our knowledge this is a first time that the issue of quantifying the impact of aging for multiple biometric modalities is treated using a unified approach. It is anticipated that the proposed work in the area will influence the design of future intelligent biometric-based HCI systems.

\section{Literature Review}

A number of surveys and books have been published on the general topic of person authentication using unimodal or multimodal biometric features [12, 13, 21, 22]. However, in those cases only limited discussion related to the effects of aging on biometric templates is presented. Similarly in surveys dedicated to person identification based on specific modalities $[2,23,25,26]$ the topic of aging is not considered in detail. An exception is a survey on iris recognition by Bowyer et al [3] where the effects of aging on iris recognition are explicitly discussed.

Few studies that aim to assess the deterioration of performance of biometric authentication over time appeared in the literature. However, in such studies usually only short term aging effects are considered. Min et al [18] conclude that as the time period between template creation and recognition increases, the performance of a face recognition system decreases dramatically. Poh at al [20] also investigate short-term aging effects on 3D face recognition. Experimental results indicate that aging affects different users in different ways, thus a proper aging invariant biometric authentication system should be user-specific.

All approaches mentioned above aim to highlight the problem of aging rather than to quantify the effects of aging. As an alternative Baker et al [1] attempt quantify the effect of aging on iris templates by comparing the hamming distance between pairs of iris images of the same subject captured with a time difference of four years. According to the results, the differences in the hamming distances recorded indicate that iris patterns may be deformed due to aging.

A limited number of studies try to investigate the use of biometric technology in Human Computer Interaction [6]. The majority of them look into the problem from the biometric perspective; the aim is either to build appropriate interfaces to increase the adoption of biometrics for security reasons [4, 8] or to create authentication systems based on users' interaction with computers in their everyday work [24]. The use of biometrics for creating user-adapted human computer interfaces only recently 
gained some attention with the creation of special tracks in some conferences [10]. Nevertheless, utilization of biometric based transactions with computers, like the fingerprint based access, for the purpose of age estimation and user profile adaptation has not been examined in other studies so far.

\section{Quantification of Aging Effects}

Let vector $\mathbf{u}_{\mathrm{i}}^{\mathrm{j}}$ be the $\mathrm{j}^{\text {th }}$ biometric template of the $\mathrm{i}^{\text {th }}$ user of a biometric based authentication system. Without loss of generality we assume that there are Ni different biometric templates for the $\mathrm{i}^{\text {th }}$ user. We can form an extended vector $\mathbf{u}_{\mathrm{i}}$ representing user $\mathrm{i}$ by concatenating vectors $\mathbf{u}_{\mathrm{i}}^{\mathrm{j}}, \mathrm{j}=1, \ldots, \mathrm{N}_{\mathrm{i}}$. The mean extended template (M) among all templates of all users in a pool is calculated and the similarity measure $s_{i}$, between user $\mathrm{i}$ and the mean template is computed using the correlation coefficient of vectors $\mathbf{u}_{\mathrm{i}}$ and $\mathbf{M}$. The homogeneity measure $(\mathrm{H})$ among all templates can be obtained by taking the average value of similarities for all users i. Similarly, a measure of dispersion (D) can be obtained by taking the standard deviation of similarities for all users i. The homogeneity measure gives an indication of the similarity between members of the distribution and dispersion gives an indication of the spread of the correlation coefficients for different users. Although it is possible to calculate the homogeneity and dispersion of a multivariate distribution using other methods, the proposed method was selected because it is standardized, enabling in that way the comparative evaluation of aging effects for different biometric modalities.

Given a set of templates belonging to a number of users, the method outlined above was used for estimating the homogeneity and dispersion among all biometric templates in a dataset. The dataset is then divided into templates corresponding to the younger and older users using a predetermined age threshold and the homogeneity and dispersion measures for each age group are also estimated. The Aging Impact (AI) of a template is defined as the difference between the homogeneity and dispersion metrics of two different groups:

$$
A I=\frac{H_{i}-H_{\text {all }}}{\sqrt{\left(\frac{D_{i}^{2}}{n_{i}}+\frac{D_{\text {all }}^{2}}{n_{\text {all }}}\right)}}
$$

where $\mathrm{H}_{\mathrm{i}}, \mathrm{D}_{\mathrm{i}}$ are the homogeneity and dispersion measures for the $\mathrm{i}^{\text {th }}$ age group while $\mathrm{H}_{\text {all }}, \mathrm{D}_{\text {all }}$ are the corresponding values computed over all database members. Similarly, $\mathrm{n}_{\mathrm{i}}$ and $\mathrm{n}_{\text {all }}$ are the number of samples in the $\mathrm{i}^{\text {th }}$ group and all samples in the database respectively. In the case that multiple age groups are considered the overall Aging Impact is the average of the Aging Impact between each group and the distribution of all samples. The largest the value of the AI measure the biggest the difference between the distributions of template values in a certain age group when compared with the distribution of all samples, leading to an increased dependency of the template to aging variation. For example if a biometric feature exhibiting a high Aging Impact factor is used in an identity verification application, it is highly probable that the system will fail to verify the identity of the user as the user grows older. Similarly the use of features with low Aging Impact in an age estimation application will result in a poor performance as the features used will not provide adequate information for discriminating between different ages. 


\section{Experimental Investigation}

The method for assessing the impact of aging presented in the previous section was tested on different modalities and different types of feature vectors, in an attempt to illustrate its applicability in quantifying the impact of aging effects.

\subsection{Experiments with Face Templates}

Experiments using face templates were carried out using samples from the FG-NET Aging Database [15]. The FG-NET Aging Database is a publicly available image database containing face images showing a number of subjects at different ages. The database contains 1002 images from 82 different subjects with ages ranging between newborns to 69 years old subjects. On average there are 12 images per subject in the database.

In this experiment we consider the use of templates constructed based on an Active Appearance Model (AAM) [5] coding scheme. Since the FG-NET aging dataset contains multiple samples per subject, the calculation of the homogeneity and dispersion measures is done for each subject individually and the overall results are calculated by taking the average values of the two measures among the 82 subjects. For the needs of the experiment face images belonging to the same subject are divided into a set of young and old. Apart from using templates representing the overall facial region, the impact of aging on the upper face, lower face, eyes and nose regions is also investigated. In order to study the effects of aging for different genders, the analysis is performed in a gender-specific style.

According to the results (see Table 1) the impact of aging varies according to the facial region considered, the age group and the gender of the users. For example in old males the impact of aging on the lower face is 22 but the impact of aging on the same region for young males is 13 . This is expected as the texture of the lower facial regions of old males undergoes dramatic changes due to the possible appearance of beards and moustaches. The results show that the most dramatically affected facial region is the eye region. Bearing in mind that the FG-NET aging dataset contains unconstrained face images, the results presented contain noisy responses that result from non-aging related variations appearing on faces. Ideally experiments using samples that mainly display aging variation need to be conducted in order to obtain definite conclusions regarding the invariance of different facial regions to aging.

Table 1. Aging Impact for different groups of samples from the FG-NET Aging Database. AIy, AIo, AI are the Aging Impact factors for the group with young samples, old samples and the overall impact respectively.

\begin{tabular}{|c|l|l|l|l|l|l|}
\hline \multirow{2}{*}{ Facial region } & \multicolumn{3}{|c|}{ Males } & \multicolumn{3}{c|}{ Females } \\
\cline { 2 - 7 } & $\mathrm{AI}_{\mathrm{y}}$ & $\mathrm{AI}_{\mathrm{o}}$ & $\mathrm{AI}$ & $\mathrm{AI}_{\mathrm{y}}$ & $\mathrm{AI}_{\mathrm{o}}$ & $\mathrm{AI}$ \\
\hline Overall face & 10 & 25 & 17 & 2 & 21 & 11 \\
\hline Upper face & 8 & 19 & 14 & 16 & 17 & 16 \\
\hline Lower face & 13 & 22 & 18 & 3 & 25 & 14 \\
\hline Nose & 12 & 30 & 21 & 7 & 17 & 12 \\
\hline Eyes & 16 & 21 & 18 & 19 & 27 & 23 \\
\hline
\end{tabular}




\subsection{Experiments with Palm and Fingerprint Templates}

Experimentation for the palm and fingerprint modalities is based on biometric templates constructed with the aid of MPEG-7 visual features [11] computed using the MPEG-7 experimentation software [19]. We have used the POLYBIO database [14] for our experiments. In this database there are four hand images per subject taken using low cost cameras as well as four fingerprint images per finger per subject taken using a light-reflection based fingerprint sensor.

Four different templates were created from every hand image, corresponding to the Color Layout (CL), the Region Shape (RS), the Edge Histogram (EH) and the Homogeneous Texture (HT) descriptors. The above descriptors were chosen to represent the three different types of visual descriptors (color, shape, texture); therefore, the palm images are not used only for computing hand geometry but the color and texture of the upper part of the palm. Each template is examined in isolation and the results are summarized in Table 2. According to the results the highest Aging Impact is recorded in Edge Histogram; that is edge information of the upper part of hand changes significantly with age. Increased aging effects on edge information are caused by reduced skin elasticity, spot appearance or enlargement and veins' pump up mainly encountered in older persons.

For the fingerprint experiments we used only the images of the left index. The templates used are the same as in the hand geometry case with the exception of Region Shape which was excluded due to the absence of any shape information in fingerprint images. According to the results, shown in Table 2, the highest Aging Impact is recorded for features based on Edge Histogram. This is something expected; fingerprint images contain a lot of edge information while aging reduces skin elasticity, and therefore proper finger fitting to the fingerprint sensor reducing in that way the amount of edge information captured by the sensor. In addition, as people get older edges on fingerprints wear out and the probability of finger injuries also increases.

However, we should take into account that both in the case of experiments with palms and fingerprints, the intra class variation (variation across subjects of the same group) is also high and may be higher than the inter class variation (variation across age) smoothing the overall Aging Impact values.

Table 2. Aging Impact for different groups of samples from the POLYBIO multimodal database. $\mathrm{AI}_{\mathrm{y}}, \mathrm{AI}_{\mathrm{o}}, \mathrm{AI}$ are the Aging Impact factors for the group with young samples, old samples and the overall impact respectively

\begin{tabular}{|c|c|c|c|c|}
\hline Modality & Template Type & $\mathbf{A I}_{\mathbf{v}}$ & $\mathbf{A I}_{\mathbf{0}}$ & $\mathbf{A I}$ \\
\hline \multirow{4}{*}{ Palm } & Scalable Color & 0.19 & 0.51 & 0.35 \\
\cline { 2 - 5 } & Region Shape & 0.60 & 0.87 & 0.73 \\
\cline { 2 - 5 } & Edge Histogram & 0.21 & 1.56 & 0.89 \\
\cline { 2 - 5 } & Homogeneous Texture & 0.22 & 0.93 & 0.57 \\
\hline \multirow{3}{*}{ Fingerprints } & Scalable Color & 0.60 & 0.03 & 0.32 \\
\cline { 2 - 5 } & Edge Histogram & 1.68 & 0.35 & 1.01 \\
\cline { 2 - 5 } & Homogeneous Texture & 0.68 & 0.55 & 0.61 \\
\hline
\end{tabular}




\subsection{Experiments with Templates Based on Hand and Face Movements}

In this experiment we consider the use of templates containing information that characterizes hand and head movements of users. In this context, volunteers who participated in this experiment were requested to use the mouse for tracing as accurately as possible the outline of a horizontal line, a rectangle and an ellipse presented on the screen. The ability of each user to trace accurately the shape was calculated by considering the mean Euclidean distance and the difference in the direction of pointer movement when compared with the target shape contour. The three measures for each volunteer and each shape (line, rectangle and ellipse) constitute a user-template. The same experimental set-up was used for assessing templates that contain features extracted from head movements. In this case a tracking algorithm suitable for locating the position of the eyes of a subject while he/she is tracing a shape on the screen was used [7].

Thirty volunteers with ages ranging from 8 years old up to 43 years old participated in the experiment. These volunteers were divided into the group of young (ages 8 to 17 ) and group of old (ages of 18 to 43 ). All volunteers who participated in the experiment were regular computer users, familiar and experienced with the operation of the mouse. Because none of the volunteers used face tracking for human computer interaction purposes before, each volunteer was allowed a five-minute training session in order to get familiar with the face tracker operation before the actual data acquisition exercise. The results obtained (see Table 3) demonstrate that the highest Aging Impact is recorded for users tracing circles, either using hand or head movements. These results are consistent with results reported in the literature [17] that demonstrate the superiority of using discrepancy features derived through a circle tracing exercise as the basis for estimating the age of a person. However, the results presented on this experiment are only indicative, due to the small sample of samples considered in the experiment.

Table 3. Aging Impact for hand and face movement based templates. $\mathrm{AI}_{\mathrm{y}}, \mathrm{AI}_{\mathrm{o}}, \mathrm{AI}$ are the $\mathrm{Ag}$ ing Impact factors for the group with young samples, old samples and the overall impact respectively

\begin{tabular}{|c|c|c|c|c|}
\hline Modality & Shape Drawn & $\mathbf{A I}_{\mathbf{y}}$ & $\mathbf{A I}_{\mathbf{0}}$ & $\mathbf{A I}$ \\
\hline \multirow{3}{*}{$\begin{array}{c}\text { Hand } \\
\text { Movements }\end{array}$} & Line & 1.26 & 1.97 & 1.62 \\
\cline { 2 - 5 } & Rectangle & 0.10 & 0.21 & 0.15 \\
\cline { 2 - 5 } & Ellipse & 4.52 & 3.63 & 4.07 \\
\hline \multirow{2}{*}{ Face } & Line & 2.52 & 0.17 & 1.35 \\
\cline { 2 - 5 } Movements & Rectangle & 2.6 & 0.38 & 1.49 \\
\cline { 2 - 5 } & Ellipse & 4.47 & 3.41 & 3.94 \\
\hline
\end{tabular}

\section{Conclusions}

We have presented a method for quantifying aging effects on biometric templates, allowing in that way the selection of appropriate features for use in specific applications. The appropriate selection of features will result in the development of robust 
systems that can be used for developing biometric-based user adaptive HCI systems. A key aspect of our work is the formulation of a generic Aging Impact measure that can be used for different types of biometric modalities and templates, allowing in that way the derivation of accurate conclusions related to the aging invariance of biometric templates. It is anticipated that the proposed work in the area will influence both the design of future age-invariant biometric authentication systems and it will also provide a standardized framework for assessing the long term permanence of biometric features.

The results obtained prove the potential of the approach in assessing the age invariance of biometric templates using a standardized method. In the future we plan to perform extended experiments that will allow the derivation of concrete results related to the aging invariance of different modalities, different features, different genders and different age groups. In order to accomplice this task we are in the process of collecting suitable multimodal biometric data from subjects with diverse ages, so that the experimentation will cover all age ranges of interests.

\section{References}

1. Baker, S.E., Bowyer, K.W., Flynn, P.J.: Empirical Evidence for Correct Iris Match Score Degradation with Increased Time-Lapse between Gallery and Probe Matches. In: Tistarelli, M., Nixon, M.S. (eds.) ICB 2009. LNCS, vol. 5558, pp. 1170-1179. Springer, Heidelberg (2009)

2. Bowyer, K.W., Chang, K., Flynn, P.: A survey of approaches and challenges in $3 \mathrm{~d}$ and multi-modal $3 d+2 d$ face recognition. Computer Vision and Image Understanding 101(1), $1-15$ (2006)

3. Bowyer, K.W., Hollingsworth, K., Flynn, P.J.: Image understanding for iris biometrics: A survey. Computer Vision and Image Understanding 110, 281-307 (2007)

4. Briggs, P., Olivier, P.L.: Biometric daemons: authentication via electronic pets. In: CHI 2008 Extended Abstracts on Human Factors in Computing Systems, pp. 2423-2432 (2008)

5. Cootes, T.F., Edwards, G.J., Taylor, C.J.: Active Appearance Models. IEEE Transactions of Pattern Analysis and Machine Intelligence 23, 681-685 (2001)

6. Coventry, L., Johnson, G.I., McEwan, T., Riley, C.: Biometrics in Practice: What Does HCI Have to Say? In: Gross, T., Gulliksen, J., Kotzé, P., Oestreicher, L., Palanque, P., Prates, R.O., Winckler, M. (eds.) INTERACT 2009. LNCS, vol. 5727, pp. 920-921. Springer, Heidelberg (2009)

7. Frangeskides, F., Lanitis, A.: Multi-Modal Contact-less Human Computer Interaction. LNBIP, pp. 405-419. Springer, Heidelberg (2007)

8. Gaboa, H., Fred, A.: A behavioral biometric system based on human-computer interaction. In: Proceedings of SPIE, pp. 381-392 (2004)

9. Germanakos, P., Tsianos, N., Lekkas, Z., Mourlas, C., Samaras, G.: Capturing essential intrinsic user behaviour values for the design of comprehensive web-based personalized environments. Computers in Human Behavior 24(4), 1434-1451 (2008)

10. ICPR 2010: 20th International Conference on Pattern Recognition, Track IV: Biometrics and Human Computer Interaction, Istanbul, Turkey (2010)

11. ISO/IEC 15938-3:2001 Information Technology - Multimedia Content Description Interface - Part 3: Visual, Ver. 1 (2001)

12. Jain, A., Ross, A., Prabhakar, S.: An Introduction to Biometric Recognition. IEEE Transactions on Circuits and Systems for Video Technology 14(1) (2004) 
13. Jain, A., Patrick, F., Ross, A. (eds.): Handbook of Biometrics. Springer, Heidelberg (2008)

14. Kounoudes, A., Tsapatsoulis, N., Theodosiou, Z., Milis, M.: POLYBIO: Multimodal Biometric Data Acquisition Platform and Security System. In: Schouten, B., Juul, N.C., Drygajlo, A., Tistarelli, M. (eds.) BIOID 2008. LNCS, vol. 5372, pp. 216-227. Springer, Heidelberg (2008)

15. Lanitis, A.: Comparative Evaluation of Automatic Age Progression Methodologies. EURASIP Journal on Advances in Signal Processing, Article ID 239480 (2008)

16. Lanitis, A.: A Survey of the Effects of Aging on Biometric Identity Verification. International Journal of Biometrics 2(1), 34-52 (2010)

17. Lanitis, A.: Age Estimation Based on Head Movements: A Feasibility Study. In: 4th International Symposium on Communications, Control and Signal Processing (2010)

18. Min, J., Flynn, P.J., Bowyer, K.W.: Assessment of time dependency in face recognition. In: Kittler, J., Nixon, M.S. (eds.) AVBPA 2003. LNCS, vol. 2688, pp. 44-51. Springer, Heidelberg (2003)

19. MPEG-7 Visual Experimentation Model (XM), Version 10.0, ISO/IEC/JTC1/SC29/WG11, Doc. N4063 (2001)

20. Poh, N., Kittler, J., Smith, R., Tena, J.R.: A Method for Estimating Authentication Performance over Time, with Applications to Face Biometrics. In: Rueda, L., Mery, D., Kittler, J. (eds.) CIARP 2007. LNCS, vol. 4756, pp. 360-369. Springer, Heidelberg (2007)

21. Ross, A., Karthik, N., Jain, A.: Handbook of Multibiometrics. Springer, Heidelberg (2006)

22. Woodward, J.D., Orlans, N.M., Higgins, P.T.: Biometrics: Identity Assurance in the Information Age. McGraw-Hill, New York (2002)

23. Yager, N., Anim, A.: Fingerprint Classification: A Review. Pattern Analysis \& Applications 7(1), 77-93 (2004)

24. Yampolskiy, R.V., Govindaraju, V.: Direct and Indirect Human Computer Interaction Based Biometrics. Journal of Computers 2(10), 76-88 (2007)

25. Yampolskiy, R.V., Govindaraju, V.: Behavioural biometrics: a survey and classification. International Journal of Biometrics 1(1), 81-113 (2008)

26. Zhao, W., Chellappa, R., Rosenfeld, A., Phillips, P.J.: Face recognition: A literature survey. ACM Computing Surveys, 399-458 (2003) 